\title{
Asymptotic Conversion Point Equations for Converted Waves Reflected from a Dipping Reflector
}

\author{
Yi-Jiun Tang, Young-Fo Chang *, and Tai-Sheng Liou \\ Institute of Applied Geophysics, National Chung Cheng University, Chiayi, Taiwan, ROC
}

Received 26 March 2008, accepted 24 July 2009

\begin{abstract}
P-S converted seismic wave exploration plays an important role in detecting complex geologic structures. In this research, we derive two new asymptotic conversion point equations for the P-S converted wave reflected from a dipping reflector. The first is a quadratic asymptotic conversion point equation of the P-S converted wave reflection from a dipping reflector (DACP equation), and the second is a linear asymptotic equation (ADACP equation). DACP and ADACP equations depend on the velocity ratio $\left(V_{P} / V_{S}\right)$ of the stratum, the offset $(X)$, the depth $(Z)$ of the conversion point, and the dip angle of the stratum. The last parameter is the most sensitive of the DACP and ADACP equations in determining the conversion point position.

The two new equations can predict the conversion point positions on a deep dipping reflector accurately and directly. The accuracy of the conversion point position at shallow depth determined by the DACP equation is better than using the ADACP equation. For a shallow conversion point, for example $Z / X=0.5$, the errors of the conversion point prediction in the horizontal distance (CP error) are less than $2 \%$ for the DACP equation, but the $\mathrm{CP}$ errors are very large for the ADACP equation. If $Z / X$ is greater than 3 , the $\mathrm{CP}$ errors of the $\mathrm{ADACP}$ equation are less than $3 \%$ and this equation is more computationally efficient than the DACP equation.
\end{abstract}

Key words: Converted wave, Conversion point, Asymptotic equation, Dipping reflector

Citation: Tang, Y. J., Y. F. Chang, and T. S. Liou, 2010: Asymptotic conversion point equations for converted waves reflected from a dipping reflector. Terr. Atmos. Ocean. Sci., 21, 639-644, doi: 10.3319/TAO.2009.07.24.01(T)

\section{INTRODUCTION}

P wave reflection seismology has been successfully applied in oil exploration in resent years. Recent applications, however, have been directed to exploring complex structures in small scale reservoirs. Although a $\mathrm{P}$ wave can be easily and efficiently generated, its resolution may not be sufficient to meet present needs. For the same frequency, an $S$ wave has a shorter wavelength and provides better vertical resolutions than those of $\mathrm{P}$ wave (Geis et al. 1990). However, it is difficult to generate an $\mathrm{S}$ wave in seismic exploration, and an $\mathrm{S}$ wave can not propagate deeply due to the absorption of the strata. But P-S converted wave exploration is expected to play an important role in improving the resolution of the image of a geologic structure by the recent progress in seismic recording system and seismic data processing. Therefore in some circumstances, a P-S

\footnotetext{
* Corresponding author

E-mail:seichyo@ccu.edu.tw
}

converted wave has a higher image resolution than a $\mathrm{P}$ wave (Tatham and Goolsbee 1984; Frasier and Winterstein 1990). The large velocity contrast between salt and sediment can generate a strong P-S converted wave that can be used to delineate the salt base more accurately than an ordinary $\mathrm{P}$ wave survey ( $\mathrm{Lu}$ et al. 2003). For shallow structures, a P-S converted wave image has a higher resolution than a $\mathrm{P}$ wave image (Garotta et al. 2003).

The conversion point $(\mathrm{CP})$ of a P-S converted wave on a horizontal interface is not at the midpoint of the offset between the source and the receiver, see Fig. 1. This causes some difficulties for the common depth point (CDP) binning. Tessmer and Behle (1988) derived two equations to delineate the trajectories of the $\mathrm{CP}$ with depth from an isotropic horizontal layer: the conversion point equation (CP equation) and the asymptotic conversion point equation (ACP equation). The former equation can predict the $\mathrm{CP}$ position exactly, and the latter can only approximate the deep CP 
position. The trajectory of the CP position with depth is hyperbolic and flattens out to a straight line when the horizontal layer becomes deep (Thomsen 1999). By using the CP and ACP equations, the common conversion point (CCP) binning can be easily implemented in the P-S converted wave seismic data processing. Chang and Tang (2005) extended the $\mathrm{CP}$ equation and derived a new quartic equation (DCP equation) for the P-S converted wave reflected from a dipping reflector. This equation can accurately predict the conversion point of the P-S converted wave reflected from a dipping reflector. An analytic solution of the $\mathrm{CP}$ equation on a dipping reflector was proposed by Yuan et al. (2006). The CP equation for the refracted P-S converted wave from a dipping reflector was derived by Tang et al. (2007).

A converted wave's DMO (dip move-out) equation can calculate the travel time of a P-S converted wave reflected from an isotropic stratum with a dipping reflector (Ikelle and Amundsen 2005). However, it can not determine the $\mathrm{CP}$ position on a dipping reflector. Using a converted wave's DMO correction in the CCP binning will result in an incorrect $\mathrm{CP}$ position on the dipping reflector. Therefore, using the DCP equation in the CCP binning can avoid this problem because the DCP equation can determine the correct $\mathrm{CP}$ position on the dipping reflector.

At present, the DCP equation, a quartic equation, is time-consuming for computation. The analytic solution of the $\mathrm{CP}$ equation on a dipping reflector proposed by Yuan et al. (2006) is complicated and computationally extensive for CCP binning. In this research, we will derive new asymptotic equations of the DCP equation for the deep reflected $\mathrm{P}-\mathrm{S}$ converted wave. These equations will be more computationally efficient and direct in resolving the CP position for the P-S converted wave reflected from a deep dipping reflector.

\section{ASYMPTOTIC CONVERSION POINT EQUA- TIONS}

In a uniform horizontal isotropic stratum, if the $\mathrm{P}$ wave is generated at the source $(S)$ and propagates downward to the bottom of the stratum, shown as Fig. 1, it reflects at the interface and converts to an upward S wave and will propagate to the receiver $(G)$. The reflection point is the CP. The ACP equation is (Tessmer and Behle 1988)

$X_{1}=\left(\frac{\gamma}{1+\gamma}\right) X$

where $\gamma$ is the velocity ratio of $\mathrm{P}$ and $\mathrm{S}$ waves $\left(V_{P} / V_{S}\right)$ of the stratum, $X$ is the offset between the source and the receiver, and $X_{1}$ is the surface distance between the source and the CP.

The ray path of the P-S converted wave reflected from a dipping reflector with a dip angle of $\theta$ is shown in Fig. 2.
The incident angle of $\mathrm{P}$ wave is $\theta_{P}$, and the reflected angle of $\mathrm{S}$ wave is $\theta_{S}$. The dashed line $(\overline{G M})$ is parallel to the dipping interface and passes through the receiver point. It intersects the $\mathrm{P}$ wave's ray path at $M$. The location of the point $O$ on $\overline{G M}$ is determined by drawing a line that is perpendicular to $\overline{G M}$ and passes through CP. The lengths of $\overline{O M}, \overline{O G}$, and $\overline{O C P}$ are $X_{11}, X_{22}$, and $Z_{1}$, respectively. $Z$ is the depth of CP. $X_{2}$ is the surface distance between the receiver and $\mathrm{CP}$, and $X=X 1+X 2$.

If a source is located at $M$, Eq. (1) can be re-written as

$X_{11}=\left(\frac{\gamma}{1+\gamma}\right)\left(X_{11}+X_{22}\right)$

Grouping $X_{11}$ to the left and dividing the entire equation by $Z_{1}$ yields

$\frac{X_{11}}{Z_{1}}=\gamma \frac{X_{22}}{Z_{1}}$

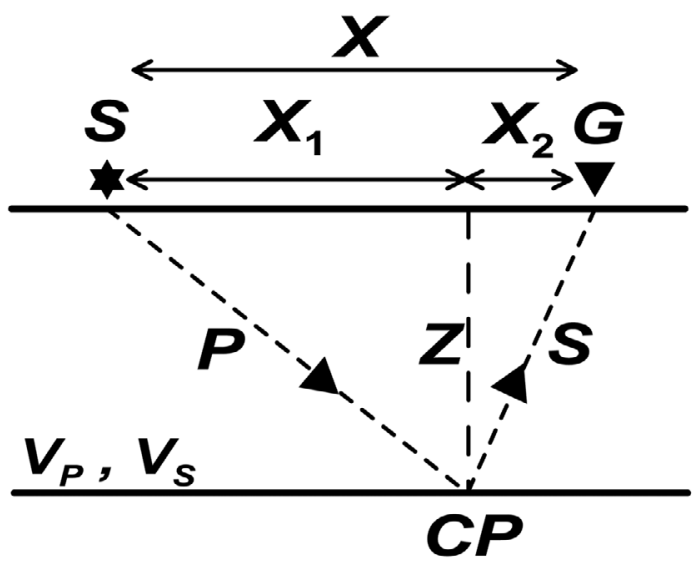

Fig. 1. Ray path of a P-S converted wave reflected from a horizontal layer. $S$ is the source, $G$ is the receiver, and $Z$ is the thickness of the layer. $\mathrm{CP}$ is the conversion point, and $X$ is the offset between the source and receiver. $X_{1}$ and $X_{2}$ are the surface distances from CP to the source and the receiver, respectively.

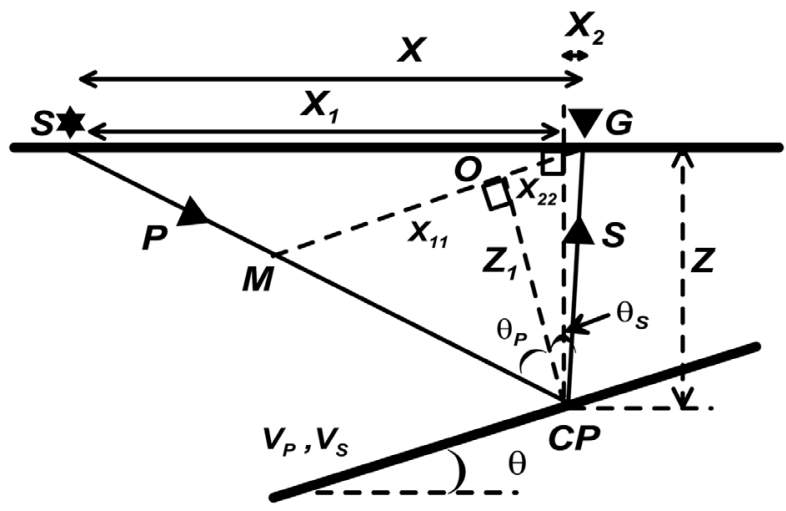

Fig. 2. Ray path of a P-S converted wave reflected from a dipping layer. $\theta$ is the dip angle of the dipping reflector. 
In terms of trigonometry, Eq. (3) can be re-written as

$$
\tan \theta_{P}=\gamma \tan \theta_{s}
$$

According to the sum and difference formulas of the trigonometric functions,

$\sin \left(\theta_{P}+\theta\right)=\sin \theta_{P} \cos \theta+\sin \theta \cos \theta_{P}=\frac{X_{1}}{\sqrt{Z^{2}+X_{1}^{2}}}$

and

$$
\cos \left(\theta_{P}+\theta\right)=\cos \theta_{P} \cos \theta-\sin \theta_{P} \sin \theta=\frac{Z}{\sqrt{Z^{2}+X_{1}^{2}}}
$$

Let $K_{1}=\sin \theta$ and $K_{2}=\cos \theta$, and $K_{1}^{2}+K_{2}^{2}=\sin ^{2} \theta+\cos ^{2} \theta$ $=1$, then Eq. (5) can be re-written as

$\sin \left(\theta_{P}+\theta\right)=K_{2} \sin \theta_{P}+K_{1} \cos \theta_{P}=\frac{X_{1}}{\sqrt{Z^{2}+X_{1}^{2}}}$

and

$$
\cos \left(\theta_{P}+\theta\right)=K_{2} \cos \theta_{P}-K_{1} \sin \theta_{P}=\frac{Z}{\sqrt{Z^{2}+X_{1}^{2}}}
$$

Solving Eq. (6) yields

$\sin \theta_{P}=\frac{K_{2} X_{1}-K_{1} Z}{\sqrt{Z^{2}+X_{1}^{2}}}$ and $\cos \theta_{P}=\frac{K_{1} X_{1}+K_{2} Z}{\sqrt{Z^{2}+X_{1}^{2}}}$

Therefore,

$$
\tan \theta_{P}=\frac{K_{2} X_{1}-K_{1} Z}{K_{1} X_{1}+K_{2} Z}
$$

Likewise, we can obtain

$$
\tan \theta_{S}=\frac{K_{2} X_{2}+K_{1} Z}{K_{2} Z-K_{1} X_{2}}
$$

Substitute Eqs. (8) and (9) into Eq. (4), then

$$
\frac{K_{2} X_{1}-K_{1} Z}{K_{1} X_{1}+K_{2} Z}=\gamma \frac{K_{2} X_{2}+K_{1} Z}{K_{2} Z-K_{1} X_{2}}
$$

Equation (10) is the asymptotic conversion point equation of the P-S converted wave on a dipping reflector (DACP equation), which is a quadratic equation of $X_{1}$. Re- placing $X_{2}$ by $X-X_{1}$, the analytic solution $\left(X_{1}\right)$ of Eq. (10) is (see Appendix A)

$$
\begin{aligned}
X_{1}= & \frac{1}{2}\left[X+\left(\frac{K_{1}^{2}-K_{2}^{2}}{K_{1} K_{2}}\right) Z\right. \\
& \left.+\sqrt{\left(\frac{Z}{K_{1} K_{2}}\right)^{2}+\frac{2 Z}{K_{1} K_{2}}\left(\frac{\gamma-1}{\gamma+1}\right) X+X^{2}}\right]
\end{aligned}
$$

Since $V_{P}>V_{S}$, then $0<\left(\frac{\gamma-1}{\gamma+1}\right)<1$. If $Z>>X$, the terms in the square root of Eq. (11), $\left(\frac{Z}{K_{1} K_{2}}\right)^{2}+\frac{2 Z}{K_{1} K_{2}}\left(\frac{\gamma-1}{\gamma+1}\right) X$ $+X^{2}$, can be approximated as $\left(\frac{Z}{K_{1} K_{2}}\right)^{2}+\frac{2 Z}{K_{1} K_{2}}\left(\frac{\gamma-1}{\gamma+1}\right) X$ $+\left(\frac{\gamma-1}{\gamma+1}\right)^{2} X^{2}$, and

$$
\begin{aligned}
& \left(\frac{Z}{K_{1} K_{2}}\right)^{2}+\frac{2 Z}{K_{1} K_{2}}\left(\frac{\gamma-1}{\gamma+1}\right) X+\left(\frac{\gamma-1}{\gamma+1}\right)^{2} X^{2} \\
& =\left[\frac{Z}{K_{1} K_{2}}+\left(\frac{\gamma-1}{\gamma+1}\right) X\right]^{2}
\end{aligned}
$$

Then, Eq. (11) can be reduced to (see Appendix B)

$$
X_{1}=\left(\frac{\gamma}{1+\gamma}\right) X+Z \tan \theta
$$

Equation (13) is the asymptotic equation of the DACP equation (ADACP equation), which is a linear equation of $X$. When the dipping reflector becomes horizontal $\left(\theta=0^{\circ}\right)$, the ADACP equation is equal to the ACP equation.

If a stratum's velocity ratio $(\gamma)$ and dip angle $(\theta)$ are 2.0 and $20^{\circ}$, respectively, the offset $(X)$ between the source and the receiver is $0.1 \mathrm{~km}$. The true positions of the $\mathrm{CP}$ position of the P-S converted wave along the dipping reflector is shown as the solid curve in Fig. 3 which is calculated by the quartic DCP equation (Chang and Tang 2005). The trajectory of the $\mathrm{CP}$ position becomes an oblique straight line at the deep depth. The hyperbolic dashed line calculated by the quadratic DACP equation is the asymptotic line of the $\mathrm{CP}$. The straight long-short dashed line is the linear approximation of the $\mathrm{CP}$, which is obtained from the ADACP equation. At the deep depth, the dash and long-short dash lines are close to the solid line, implying that the DCP equation can be approximated by the DACP and ADACP equations. However at a shallow depth, the ADACP equation has larger error than the DACP equation.

The DACP and ADACP equations are derived from the ACP equation, so they have the same assumption. When the depth of the horizontal (or dipping) reflector is greater than the offset (Depth >> Offset), the ACP, DACP, and ADACP equations can predict the conversion point accurately. 


\section{DISCUSSION}

The ACP equation is a function of the velocity ratio $\left(V_{P} / V_{S}\right)$ of the stratum and the offset (source-receiver). The DACP and ADACP equations are derived from the ACP equation. Therefore, these two new equations depend not only on $V_{P} / V_{S}$ and offset, but also on the dip angle and depth of the stratum. For a dipping stratum with dip angle of $30^{\circ}$, the errors of the conversion points in the horizontal distance $\left(\mathrm{CP}\right.$ errors $\left.=\left|C P_{\text {exact-solution }}-C P_{D A C P \text {-or-ADACP }}\right| / C P_{\text {exact-solution }}\right)$ of the DACP and ADACP equations with different $\gamma\left(V_{P} / V_{S}\right)$ are shown in Fig. 4. The CP errors of the DACP and ADACP equations go to zero when the depth-offset ratio $(Z / X)$ is large, but they become large if $Z / X$ is small. The $\mathrm{CP}$ error of the DACP equation seems to be insensitive to $\gamma$. But for the ADACP equation, greater $\gamma$ has smaller $\mathrm{CP}$ error. The $\mathrm{CP}$ errors of the ADACP equation for any $\gamma$ are always greater than those of the DACP equation. Thus, the DACP and ADACP equations can predict the $\mathrm{CP}$ position very well at the deep depth. The CP errors of the DACP equation are smaller than those of the ADACP equation, especially at a shallow depth.

We are also interested in knowing how the dip angle of the reflector affects the $\mathrm{CP}$ error of the DACP and ADACP equations. For a dipping stratum with $\gamma=2.0$, the $\mathrm{CP}$ errors of the DACP and ADACP equations with different $\theta$ are shown in Fig. 5. This result is similar to that in Fig. 4. The $\mathrm{CP}$ errors of the DACP and ADACP equations approach to zero when $Z / X$ is large, but they become large as $Z / X$ is small. Except that, when $\theta$ is very large (for example $\theta=80^{\circ}$ ), the $\mathrm{DACP}$ equation can calculate the $\mathrm{CP}$ position very precisely for any $Z / X$ values. The greater $\theta$ has smaller $C P$ error for the two equations. The $\mathrm{CP}$ errors of the ADACP equation for any $\theta$ are always greater than those of the DACP equation. In addition, both the DACP and ADACP equations are more sensitive to $\theta$ than to $\gamma$ in terms of the $\mathrm{CP}$ errors.

The CP errors of the DACP and ADACP equations will reduce to zero when $Z / X$ is large despite the values of $\theta$ and $\gamma$. In addition, when $Z / X=0.5$ and $\theta>30^{\circ}$, the $\mathrm{CP}$ errors of the DACP equation are less than $2 \%$, but the CP errors of the ADACP equation are approximately $18 \%$. Nevertheless, the $\mathrm{CP}$ errors of the ADACP equation will be less than $3 \%$ if the $Z / X$ is greater than 3 . Therefore, for doing the CCP binning of the "deep" reflected converted wave, it is more timesaving if the linear ADACP equation is used. The DACP equation can be used for the "shallow" seismic data because the $\mathrm{CP}$ errors of the DACP equation are less than $2 \%$ if the $Z / X$ is greater than 0.5 and the $\theta$ is greater than $10^{\circ}$.

Yuan et al. (2006) proposed the analytic solution of the converted wave reflected from a dipping reflector which is an exact solution of the DCP equation. But too many variables are needed in their equation. Although the DACP and $\mathrm{ADACP}$ equations are asymptotic equations and there are some errors in the conversion point position estimation at a shallow depth, when comparing the other errors which result from the trace binning for the P-S converted wave seismic data processing, those particular errors are quite small.

\section{CONCLUSION}

We have derived the DACP and ADACP equations

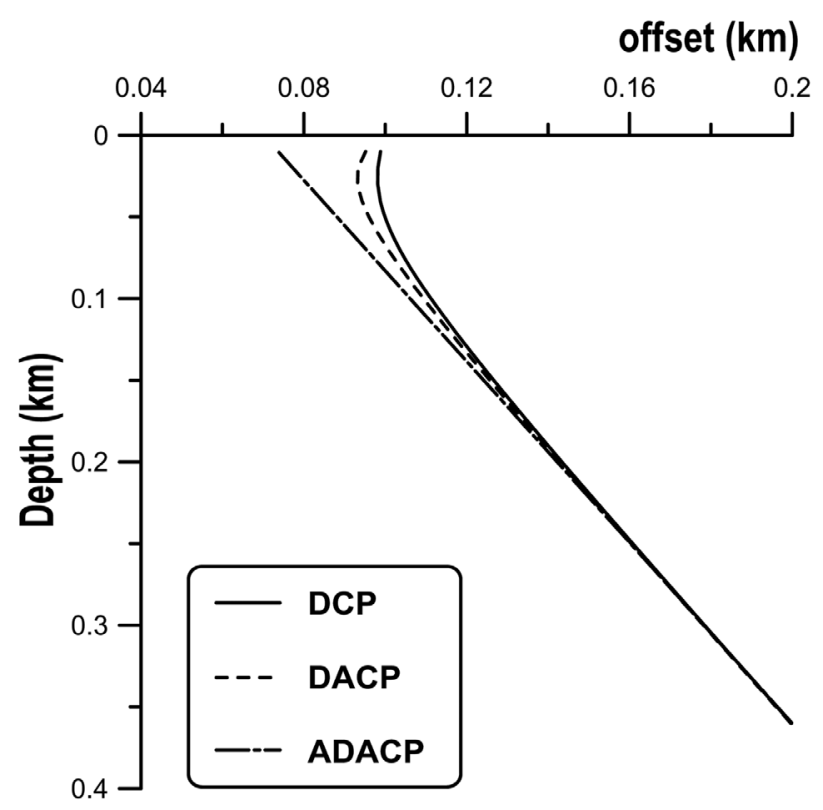

Fig. 3. The trajectories of the CP with depth for the DCP, DACP, and ADACP equations.

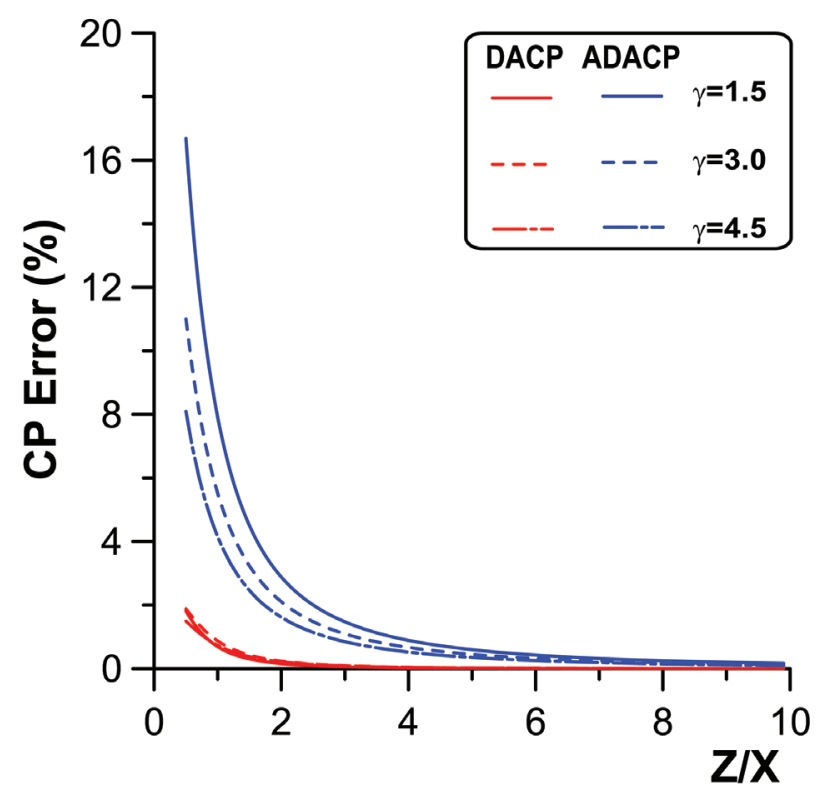

Fig. 4. The relationships of the errors of the conversion point in the horizontal distance (CP error) vs. the depth-offset ratio $(Z / X)$ for DACP and ADACP equations with three velocity ratios $(\gamma=1.5,3.0$, and 4.5). The dip angle $(\theta)$ of the dipping reflector is $30^{\circ}$. 


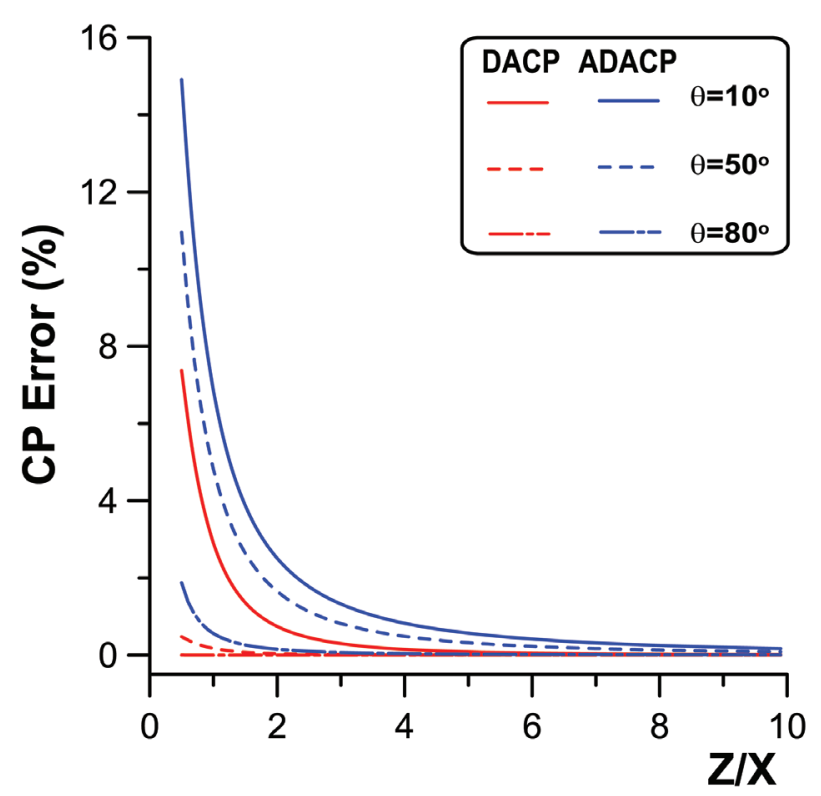

Fig. 5. The relationships of the errors of the conversion point in the horizontal distance (CP error) vs. the depth-offset ratio $(Z / X)$ for $\mathrm{DACP}$ and $\mathrm{ADACP}$ equations with three dip angles $\left(\theta=10^{\circ}, 50^{\circ}\right.$, and $\left.80^{\circ}\right)$. The velocity ratio $(\gamma)$ of the stratum is 2 .

from the ACP equation for the P-S converted wave reflected from a dipping reflector. The DACP equation is a quadratic equation and the ADACP is a linear equation. Therefore, in estimating the $\mathrm{CP}$ position of $\mathrm{P}-\mathrm{S}$ converted wave using the $\mathrm{DACP}$ and ADACP equations is faster than using the DCP equation which is useful for the CCP binning and move-out correction of the P-S converted wave reflected from a dipping reflector. These new equations depend on the velocity ratio of the stratum, the offset between the source and receiver, the dip angle and depth of the dipping reflector. The dip angle is the dominant factor of the DACP and ADACP equations in determining the $\mathrm{CP}$ position. Therefore, the success of using the DACP and ADACP equations in the $\mathrm{P}-\mathrm{S}$ converted wave data processing relies heavily on the accuracy of determining the dip angle of the stratum.

Acknowledgement We are grateful to Prof. Richard Gibson and one anonymous reviewer for their helpful suggestions, which have largely improved our manuscript. This research was financially supported by the National Science Council under grant no. NSC972116M194005.

\section{REFERENCES}

Chang, Y. F. and Y. J. Tang, 2005: The equation of conversion point for the converted wave on a dipping layer. SEG Expanded Abstracts, 24, 1007-1009, doi: 10.1190/1.2147849. [Link]

Frasier, C. and D. Winterstein, 1990: Analysis of conven- tional and converted mode reflections at Putah sink, California using three-component data. Geophysics, 55, 646-659, doi: 10.1190/1.1442877. [Link]

Garotta, R. and P. Y. Granger, 2003: Acquistion/Processing - Some requirements for PS-mode acquisition. The Leading Edge, 22, 106-112, doi: 10.1190/1.1559036. [Link]

Geis, W. T., R. R. Stewart, M. J. Jones, and P. E. Katopodis, 1990: Processing, correlating, and interpreting converted shear waves from borehole data in southern Alberta. Geophysics, 55, 660-669, doi: 10.1190/1.1442878. [Link]

Ikelle, L. T. and L. Amundsen, 2005: Introduction to petroleum seismology, Society of Exploration Geophysicists, Tulsa, Oklahoma, 679 pp, ISBN: 1-56080-129-8.

Lu, R. S., D. E. Willen, and I. A. Watson, 2003: Identifying, removing, and imaging P-S conversions at saltsediment interfaces. Geophysics, 68, 1052-1059, doi: 10.1190/1.1581076. [Link]

Tang, Y. J., K. C. Yang, and Y. F. Chang, 2007: The conversion-point equation for the refracted converted PSwave on a dipping layer. SEG Expanded Abstracts, 26, 1064-1068, doi: 10.1190/1.2792586. [Link]

Tatham, R. H. and D. V. Goolsbee, 1984: Separation of $\mathrm{S}$-wave and P-wave reflections offshore western Florida. Geophysics, 49, 493-508, doi: 10.1190/1.1441685. [Link]

Tessmer, G. and A. Behle, 1988: Common reflection point data-stacking technique for converted waves. Geophys. Prospect.,36, 671-688, doi: 10.1111/j.1365-2478.1988. tb02186.x. [Link]

Thomsen, L., 1999: Converted wave reflection seismology over inhomogeneous, anisotropic media. Geophysics, 64, 678-690, doi: 10.1190/1.1444577. [Link]

Yuan, C., S. Peng, and C. Li, 2006: An exact solution of the conversion point for the converted waves from a dipping reflector with homogeneous (isotropic) overburden. Geophysics, 71, T7-T11, doi: 10.1190/1.2149900. [Link]

\section{APPENDIX A}

Equation (10) is

$\frac{K_{2} X_{1}-K_{1} Z}{K_{1} X_{1}+K_{2} Z}=\gamma \frac{K_{2} X_{2}+K_{1} Z}{K_{2} Z-K_{1} X_{2}}$

Replacing $X_{2}$ by $X-X_{1}$, Eq. (A-1) can be re-written as

$\frac{K_{2} X_{1}-K_{1} Z}{K_{1} X_{1}+K_{2} Z}=\gamma \frac{K_{2}\left(X-X_{1}\right)+K_{1} Z}{K_{2} Z-K_{1}\left(X-X_{1}\right)}$

Expanding Eq. (A-2) gets 
$K_{2}^{2} X_{1} Z-K_{1} K_{2} X_{1} X+K_{1} K_{2} X_{1}^{2}-K_{1} K_{2} Z^{2}+K_{1}^{2} X Z-K_{1}^{2} X_{1} Z-$

$\gamma K_{1} K_{2} X X_{1}+\gamma K_{1} K_{2} X_{1}^{2}-\gamma K_{1}^{2} X_{1} Z-\gamma K_{2}^{2} X Z+\gamma K_{2}^{2} X_{1} Z-$

$\gamma K_{1} K_{2} Z^{2}=0$

Arranging Eq. (A-3),

$(1+\gamma) K_{1} K_{2} X_{1}^{2}+\left[(1+\gamma)\left(K_{2}^{2}-K_{1}^{2}\right) Z-(1+\gamma) K_{1} K_{2} X\right] X_{1}+$

$\left[-(1+\gamma) K_{1} K_{2} Z^{2}+\left(K_{1}^{2}-\gamma K_{2}^{2}\right) X Z\right]=0$

Dividing Eq. (A-4) by $(1+\gamma) K_{1} K_{2}$ then yields

$X_{1}^{2}+\left(\frac{K_{2}^{2}-K_{1}^{2}}{K_{1} K_{2}} Z-X\right) X_{1}+\left[\frac{K_{1}^{2}-\gamma K_{2}^{2}}{(1+\gamma) K_{1} K_{2}} X Z-Z^{2}\right]=0$

Let

$a=1, b=\frac{K_{2}^{2}-K_{1}^{2}}{K_{1} K_{2}} Z-X, c=\frac{K_{1}^{2}-\gamma K_{2}^{2}}{(1+\gamma) K_{1} K_{2}} X Z-Z^{2}$

Then,

$X_{1}=\frac{-b \pm \sqrt{b^{2}-4 a c}}{2 a}$

For the P-S converted wave,

$X_{1}=\frac{-b+\sqrt{b^{2}-4 a c}}{2 a}$

Then,

$b^{2}-4 a c=\frac{\left(K_{2}^{2}+K_{1}^{2}\right)^{2}}{K_{1}^{2} K_{2}^{2}} Z^{2}-\frac{2\left(K_{2}^{2}+K_{1}^{2}\right)}{K_{1} K_{2}}\left(\frac{1-\gamma}{\gamma+1}\right) X Z+X^{2}$

Since $K_{1}=\sin \theta$ and $K_{2}=\cos \theta$, Eq. (A-9) can be re-written

as

$b^{2}-4 a c=\left(\frac{Z}{K_{1} K_{2}}\right)^{2}+\frac{2 Z}{K_{1} K_{2}}\left(\frac{\gamma-1}{\gamma+1}\right) X+X^{2}$

Therefore, the solution of the Eq. (A-5) is

$$
\begin{aligned}
X_{1}= & \frac{1}{2}\left[X+\left(\frac{K_{1}^{2}-K_{2}^{2}}{K_{1} K_{2}}\right) Z\right. \\
& \left.+\sqrt{\left(\frac{Z}{K_{1} K_{2}}\right)^{2}+\frac{2 Z}{K_{1} K_{2}}\left(\frac{\gamma-1}{\gamma+1}\right) X+X^{2}}\right]
\end{aligned}
$$

Equation (A-11) is the solution of the DACP equation.

\section{APPENDIX B}

Since $V_{P}>V_{S}$, then $0<\left(\frac{\gamma-1}{\gamma+1}\right)<1$. If $Z>>X$, the terms in the square root of Eq. (11), $\left(\frac{Z}{K_{1} K_{2}}\right)^{2}+\frac{2 Z}{K_{1} K_{2}}\left(\frac{\gamma-1}{\gamma+1}\right) X$ $+X^{2}$, can be approximated as $\left(\frac{Z}{K_{1} K_{2}}\right)^{2}+\frac{2 Z}{K_{1} K_{2}}\left(\frac{\gamma-1}{\gamma+1}\right) X$ $+\left(\frac{\gamma-1}{\gamma+1}\right)^{2} X^{2}$ which is exactly the expansion of $\left[\frac{Z}{K_{1} K_{2}}+\right.$ $\left.\left(\frac{\gamma-1}{\gamma+1}\right) X\right]^{2}$. Then,

$$
X_{1}=\frac{1}{2}\left\{X+\left(\frac{K_{1}^{2}-K_{2}^{2}}{K_{1} K_{2}}\right) Z+\sqrt{\left[\frac{Z}{K_{1} K_{2}}+\left(\frac{\gamma-1}{\gamma+1}\right) X\right]^{2}}\right\}
$$

$$
\begin{aligned}
& =\frac{1}{2}\left[X+\left(\frac{K_{1}^{2}-K_{2}^{2}}{K_{1} K_{2}}\right) Z+\frac{Z}{K_{1} K_{2}}+\left(\frac{\gamma-1}{\gamma+1}\right) X\right] \\
& =\frac{1}{2}\left(\frac{2 \gamma}{\gamma+1} X+\frac{K_{1}^{2}-K_{2}^{2}+1}{K_{1} K_{2}} Z\right)
\end{aligned}
$$

Since $K_{1}=\sin \theta$ and $K_{2}=\cos \theta$, Eq. (B-3) can be re-written as

$$
\begin{aligned}
X_{1} & =\frac{1}{2}\left[\frac{2 \gamma}{\gamma+1} X+\frac{K_{1}^{2}-K_{2}^{2}+\left(K_{1}^{2}+K_{2}^{2}\right)}{K_{1} K_{2}} Z\right] \\
& =\frac{1}{2}\left(\frac{2 \gamma}{\gamma+1} X+\frac{2 K_{1}^{2}}{K_{1} K_{2}} Z\right) \\
& =\frac{\gamma}{\gamma+1} X+\frac{K_{1}}{K_{2}} Z \\
& =\frac{\gamma}{\gamma+1} X+Z\left(\frac{\sin \theta}{\cos \theta}\right) \\
& =\frac{\gamma}{\gamma+1} X+Z \tan \theta
\end{aligned}
$$

